Original Research Article

\title{
A study of adverse drug reaction profile of tuberculosis patients attending DOTS center at Dr. Bhim Rao Ambedkar memorial hospital, Raipur, Chhattisgarh, India
}

\author{
Anand Rao, Tabish Ahmed*, Tanveer A. Khan, N. B. Chandel, Chandresh Mishra
}

Department of Pharmacology, Government Medical College, Rajnandgaon, Chhattisgarh, India

Received: 13 December 2018 Accepted: 08 March 2019

*Correspondence to:

Dr. Tabish Ahmed,

Email:

Dr.tabishahmed@gmail.com

Copyright: (C) the author(s), publisher and licensee Medip Academy. This is an openaccess article distributed under the terms of the Creative Commons Attribution NonCommercial License, which permits unrestricted noncommercial use, distribution, and reproduction in any medium, provided the original work is properly cited.

\begin{abstract}
Background: Tuberculosis is second leading cause of death in the world. The causative organism is Mycobacterium tuberculosis. The objective was to study the adverse reactions of the patients attending the DOTS center and to assess their causality and severity of reported ADRs.

Methods: Present study was a prospective observational study carried at the DOTS center of Dr. Bhim Rao Ambedkar Memorial Hospital, Raipur, Chhattisgarh, India between August 2011 to July 2012 (One year). The patients were monitored for adverse drug reactions. The assessment of ADRs were based upon the WHO assessment scale, Naranjo scale, European A.B.O scale.

Results: Total number of patients attending DOTS center was 816 . The number of males (428) exceeded that of females (388). Majority of patients in this study belonged to $21-30$ years $(26.96 \%)$ next $31-40$ years $(25.24 \%)$ and $41-50$ years $(16.5 \%)$ of age group. Prevalence of ADRs were more in males $(57 \%)$ than in females $32(43 \%)$. Majority of ADRs reported were moderate $33(35.22 \%)$ followed by $29(46,77 \%)$ were mild, no severe ADRs reported. According to severity of ADRs seen were gastritis $28(45 \%)$ followed by $10(16 \%)$ rashes , 10 $(16,12 \%)$ of arthralgia, $3(4.83 \%)$ of hepatitis, $6(9.7 \%)$ of peripheral neuropathy, $2(3 \%)$ onsets of ADRs after starting anti-tubercular drug were $12(19.35 \%)$ in 0 1 week followed by 19 (30\%) ADRs showed onset in 1-2 week and 2-3 week, 8 $(13 \%)$ in 3-4 week $3(5 \%)$ in 4-5 week and 1 (2\%) in 5-6 week.

Conclusions: The casual link between the ADRs and the suspected antitubercular drug by Naranjo scale definitely relationship was established between the anti-tubercular drug and ADRs in 7 (11.25\%) patient while $22(35.45 \%)$ probable and $33(53.22 \%)$ ADRs were categorized as possible.
\end{abstract}

Keywords: Adverse Drug reaction, Antitubercular Drugs, DOTS, Naranjo scale

\section{INTRODUCTION}

Adverse drug reactions are of great concern to the general public, the pharmaceutical industry, the regulatory authorities and the medical profession.

According to $\mathrm{WHO}$, an adverse drug reaction is any response of drug that is noxious and unintended, that occur at dose normally used in human for prophylaxis, Diagnosis and treatment of diseased or for the modification of physiological function. ADRs are common occurrence in hospital settings, more so it the commonly and is attributed is the severely and complexly of the disease process use of multiple drugs and drug interactions. ${ }^{1}$

The Worlds Health Organization (WHO) declared tuberculosis (TB) as a global emergency in $1993 .^{2}$ The WHO estimated that globally 9.2 million new cases of TB occurred in 2007 (139/100,00 population) India, China, Indonesia, Nigeria and South Africa ranks first to fifth in 
the total number of incident cases. MDR-TB and XDR-TB are strong indicators of TB control programme failures due to multi drug therapy and their ADR. The global estimate of the burden of MDR-TB (511,000 incident case, 150,000 deaths) and XDR-TB, cases (50,000 cases, 30,000 death). ${ }^{3}$

Before the advent of the DOTS programme, high prevalence countries like India had a National Tuberculosis Programme (NTP) started in 1962 (NTP employs a daily regimen of anti-TB drugs) as a truly integrated programme, implemented through District Tuberculosis Centers (DTCs) and peripheral health institution. ${ }^{4}$ In order to intensify the efforts to control TB, the Government of India gradually replaced NTP by the DOTS strategy/programme in 1993 and it is now known as the revised National Tuberculosis programme (RNTCP). The objective of this revised strategy was to achieve a cure rate of $85 \%$ for infections and seriously ill patient through intermittent (three days a week) supervised short course chemotherapy are the directly observed treatment short course (DOTS). ${ }^{5}$

Anti-tubercular drugs, just like other drugs used in clinical practice, are not free from ADRs. The added problem is that combinations of drugs are always used for prolonged periods of time therefore, it is likely that the adverse reactions of one drug may be potentiated by the companion drugs used. Moreover, the adverse drug reaction (ADRs) to the drugs used is one of the major reasons for the patient default for treatment. A general knowledge of the various ADRs and their management is essential for the effective management of TB. ${ }^{6}$ All anti-tubercular drugs can cause adverse drug reactions and may result in ADRs involving almost all system in body, including the gastrointestinal tract, liver skin, nervous system, vestibular apparatus and the eyes. Numerous clinical trials have determined that there is a $15 \%$ probability of an adverse effect occurring in a patient who is on a multiple anti tubercular drug regimen and adverse reactions mostly tend to occur in the first three months of treatment.

Causality assessment of ADRs is the structured and standardized assessment of individual patients/case report of the likelihood of involvement of suspected drug in causality assessment included the complete knowledge about clinical event, baseline symptoms of the patient, medication details, complete medical history of the patient, possible drug interactions and outcomes of the reaction upon de-challenge and/or re-challenge. There are many systems used to assess the subject of causality relationships. ${ }^{7}$

$\begin{array}{ll}\text { - } & \text { WHO assessment scale, } \\ \text { - } & \text { Naranjo scale, } \\ \text { - } & \text { European scale, } \\ \text { - } & \text { Karch and lasagna scale, } \\ \text { - } & \text { Kramer scale, } \\ \text { - } & \text { Yayesian neural network, } \\ \text { - } & \text { Spanish quantitative imputation system. }\end{array}$

\section{WHO assessment scale}

Various criteria required for assessment of causality includes knowledge about nature of the reaction, any temporal time relationship with the event, previous reports on reported reaction, de-challenge, re-challenge and other possible alternate causes. The causal relationships have been categorized as certain, probable, possible, unlikely, conditional and un-assessable.

\section{WHO categories}

\section{Certain}

A clinical event, including laboratory test abnormality, occurring in a plausible time relationship to drug administration and which cannot be explained by concurrent disease or other drugs or chemicals. The event must be definitive pharmacologically or phenomenologically, using a satisfactory re-challenge procedure if necessary.

\section{Probable}

A clinical event, including laboratory test abnormality, with a reasonable time sequence to administration of the drug, unlikely to be attributed to concurrent or other drugs or chemicals and which follow a clinically reasonable response on withdrawal (de-challenge). Re-challenge information is not required to fulfil this definition.

\section{Possible}

A clinical event, including laboratory test abnormality, with a reasonable time sequence to administration of the drug but which could also be explained by concurrent disease or other drugs or chemicals. Information on drug withdrawal may be lacking or unclear.

\section{Unlikely}

A clinical event including laboratory test abnormality, with a temporal relationship to drug administration, which makes causal relationship improbable and in which other drug, chemical or underlying disease provide plausible explanations.

\section{Conditional/unclassified}

A clinical event including laboratory test abnormality, reported as an adverse reaction about which more data is essential for a proper assessment or the additional data are under examination.

\section{Un-assessable/Unclassifiable}

A report suggesting an adverse reaction, which cannot be judged because information is insufficient or contradictory and which cannot be supplemented or verified. ADRs categorized under certain category shows definite 
relationship between the drug and the drug and the event e.g., penicillin anaphylaxis. In probable relationship, the definition is less stringent than for certain and does not necessitate prior knowledge of drug characteristics or clinical adverse phenomena. As stated, no re-challenge information is needed but confounding drug administration underlying disease must be absent. Causality is termed possible when relationship can neither be confirmed nor denied. Unlikely is intended to be used when the exclusion of drug causality of a clinical event seems most plausible. ${ }^{8}$

\section{Naranjo scale}

The Naranjo algorithm was developed by Naranjo et al, to overcome some of the limitation of standardized causality assessment so as to increase the flexibility and ability to consider multiple causes. This method combines information regarding the adverse event and details specific to the particular case. It calculates the probability in favour of a specific drug cause, based on background information (e.g. epidemiological) and case information (e.g. temporal relationship).

The total score calculated from this table defines the probability category as:

- Possibly: total score 1-4,

- Probably: total score 5-8,

- Definitely: total score $>9$,

- Unlikely: total score $<0$.

A major limitation of Naranjo algorithm is its inability to do causality if re-challenging and use of placebo is not done at the time of an ADR. Also, mention about the alternative causes is essential. ${ }^{9}$

\section{European $\mathrm{ABO}$ system}

As a step towards harmonization in drug regulation in the countries of the European Union, the EU pharmacovigilance working parties proposed the following three causality categories.

- Category A- Probable,

- Category B- Possible,

- Category 0- Unclassified.

\section{Category A}

Reports including good reason and sufficient documentation to assume a causal relationship in the sense of plausible, conceivable, likely but not necessary highly probable.

\section{Category $B$}

Reports containing sufficient information to accept the possibility of a causal relationship, in the sense of not possible and not likely although the connection is uncertain and may be even doubtful example of missing data, insufficient evidence or the possibility of another expiation.

\section{Category $O$}

Reports where causality is, for one or another reason, not assessable e.g., because of missing or conflicting data. Various advantages of causality analysis include decrease in disagreement between the assessors, help in classifying uncertainly marks the individual case report and improvement of the scientific basis of causality assessment.

Limitations during causality analysis involves failure in distinguishing valid from invalid cases, occasional inability to prove the connection between a drug and an event and failure in quantifying the contribution of a drug to the development of an adverse event.

Kramer listed 15 factors interfering with the establishment of ADR causality. Some of the important factors complicating the establishment of causality of ADRs are the recently introduced drugs about whom much is not known, polypharmacy e.g., co-administration of number of AMAs with analgesics and other drugs, sudden drug withdrawal without the knowledge of treating physician and noncompliance and various non-drug therapies which are undertaken by the patient on his own. Sometimes, the patient may be suffering from other intercurrent disease that has forgotten to mention and the ADR may mimic such disease.

It is often difficult to estimate the alternative cause for suspected reaction and often many of the symptoms attributed to ADR occurs in healthy individuals taking no medications.

\section{METHODS}

The study was undertaken in the Department of Pharmacology pt. JNM Medical College, Raipur, Chhattisgarh, India. The cases included all the patients visiting the DOTS Center in one year (August 2011-July 2012) and those admitted in the medical ward Dr. Bhim Rao Ambedkar Memorial Hospital, Raipur, Chhattisgarh, India with suspected ADRs due to anti tubercular drugs. Information of the ADRs was data based was collected from DOTS Center with the help of treating physician and other care professionals in a specialized Proforma. The assessment of ADRs done with the help of following scales like WHO assessment scale, Naranjo scale, European A.B.O scale.

\section{Inclusion criteria}

- $\quad$ Patients of all the categories of TB with ADR to antitubercular agents visiting at the DOTS Center Dr. Bhim Rao Ambedkar Memorial Hospital, Raipur, Chhattisgarh, India, 
- Patients with ADR to anti-tubercular agents in ward,

- Patients above 12 year of age,

- Patient receiving minimum one anti tubercular drug.

\section{Exclusion criteria}

- Patients below 12 year of age,

- Patients who were HIV positive,

- Pregnancy,

- Patients known case of DM,

- Patients of MDR-TB and XDR-TB.

Information of patients was reviewed as details concerning the patient's age, sex, occupation, personal history, history of present illness, details of all medications taken, medication, daily dose, date of starting, stopping the medication and date of onset of suspected ADR, significant past history and the details of medications the patients were taking for any other illness and clinical examination finding with special reference to the ADRs, diagnosis and management severity of the ADR and outcomes of the treatment and sequel.

\section{RESULTS}

Total number of patients treated with DOTS therapy where 816. Out of $816,716(88 \%)$ patients were from OPD and $100(12 \%)$ were from IPD. It was $7.5 \%$ is similar to a study Dhingra et al, observed $8.37 \%$ and Marra $\mathrm{F}$ et al, found $7.3 \%$ (Figure 1). ${ }^{10,11}$

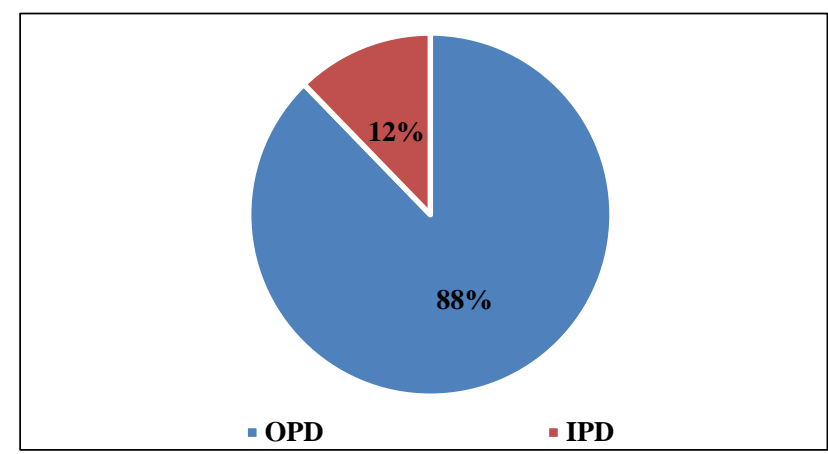

Figure 1: Department wise distribution of patients treated with DOTS.

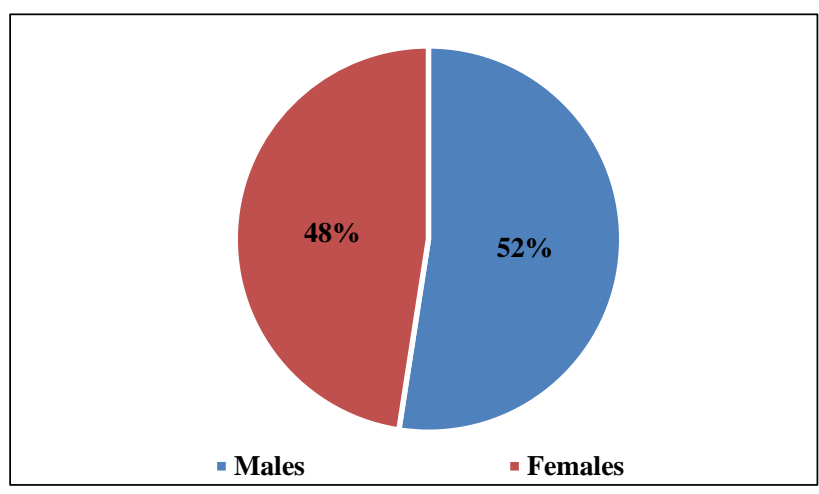

Figure 2: Serum creatinine before surgery.
The number of male patients were 428 whereas females were 388. Similar results were depicted in the study, where males were 49 as compared to 22 females (Figure 2).

Majority of patients in this study belonged to age group 2130 years (26.96\%), 31-40 years (25.24\%) and $41-50$ years $(16.5 \%)$ as also depicted in the study.

The prevalence was of ADRs was more in males (57\%) as compared to females (43\%). $80.65 \%$ of the ADRs were detected in OPD patients whereas $19.35 \%$ were found in IPD (Table 1).

Table 1: Age and gender wise prevalence of patients.

\begin{tabular}{|lll|ll|}
\hline $\begin{array}{l}\text { Age } \\
\text { group }\end{array}$ & Male & Female & Total & $\%$ \\
\hline $12-20$ & 53 & 50 & 113 & 13.89 \\
\hline $21-30$ & 116 & 104 & 220 & 26.96 \\
\hline $31-40$ & 107 & 99 & 206 & 25.24 \\
\hline $41-50$ & 73 & 68 & 131 & 16.05 \\
\hline $51-60$ & 60 & 51 & 111 & 13.60 \\
\hline$>60$ & 19 & 16 & 35 & 16.54 \\
\hline Total & $428(52.45 \%)$ & $388(47.54 \%)$ & 816 & 100.00 \\
\hline
\end{tabular}

Majority of ADRs reported were moderate $35.22 \%$ followed by $46.77 \%$ were mild, no severe ADRs reported (Figure 3).

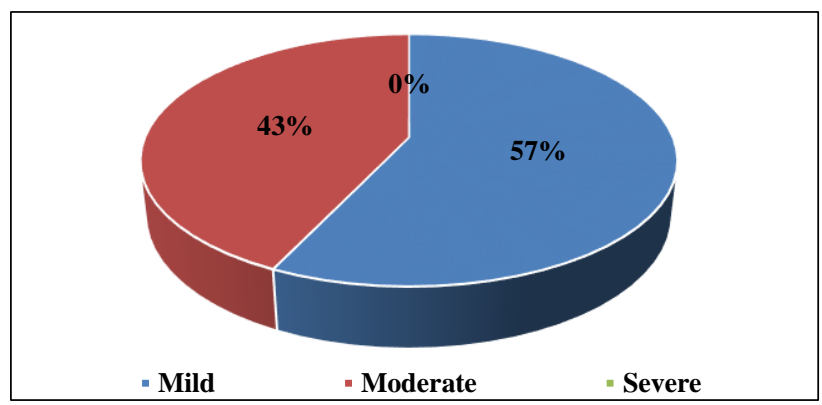

Figure 3: Severity of ADRs reported at the DOTS Center.

Table 2: Percentage wise representation of ADR reported.

\begin{tabular}{|lll|}
\hline ADR & Number & Percentage \\
\hline Gastritis & 28 & 44.82 \\
\hline Rashes & 10 & 16.12 \\
\hline Arthralgia & 10 & 16.12 \\
\hline Hepatitis & 03 & 4.83 \\
\hline Peripheral neuropathy & 03 & 4.83 \\
\hline Vertigo & 06 & 9.67 \\
\hline Flu like syndrome & 02 & 3.22 \\
\hline
\end{tabular}

According to severity of ADRs seen were gastritis $44.82 \%$, followed by rashes $16.12 \%$, arthralgia $16.12 \%$, hepatitis $4.83 \%$, peripheral neuropathy $9.67 \%$, vertigo $4.83 \%$ and flu like syndrome $3 \%$ (Table 2 ). 
Onsets of ADRs after starting anti-tubercular drug were in 0-1 week (19.35\%) followed by 1-2 week and 2-3 week (30\%), 3-4 week (13\%) in 4-5 week (5\%) and in 5-6 week (2\%) (Figure 4).

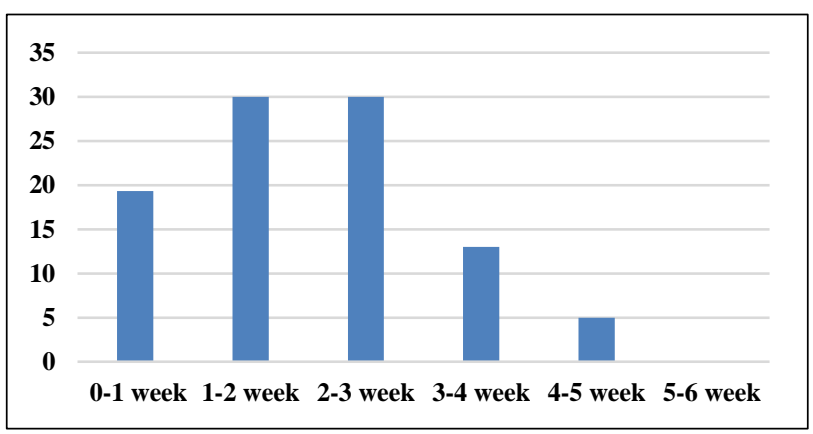

Figure 4: Week wise onset of ADR after antitubercular drug treatment.

The casual link between the ADRs and the suspected antitubercular drug by Naranjo scale definitely relationship was established between the anti-tubercular drug and ADRs in $7(11.25 \%)$ patient while $22(35.45 \%)$ probable and $33(53.22 \%)$ ADRs were categorized as possible (Table 3).

Table 3: Causality assessment of ADR according to Naranjo scale.

\begin{tabular}{|lllll|}
\hline $\begin{array}{l}\text { Anti- } \\
\text { TB } \\
\text { drug }\end{array}$ & $\begin{array}{l}\text { No. of } \\
\text { ADR }\end{array}$ & Definite & Probable & Possible \\
\hline H & 26 & 3 & 10 & 14 \\
\hline R & 16 & 0 & 6 & 10 \\
\hline Z & 10 & 1 & 3 & 4 \\
\hline E & 7 & 0 & 3 & 4 \\
\hline S & 3 & 3 & 0 & 1 \\
\hline \multirow{2}{*}{ Total } & 62 & 7 & 22 & 33 \\
& $(100 \%)$ & $(11.29 \%)$ & $(35.485 \%)$ & $(53.22 \%)$ \\
\hline
\end{tabular}

Total number of ADRs of individual drug (anti-tubercular) $\mathrm{H}(41.93 \%)$ patient reported were $(25.8 \%)$ reported to $\mathrm{R}$, (16.12\%) patient reported to $\mathrm{P}, 7 \%, 3 \%$ patients reported to $\mathrm{E}$ and $\mathrm{S}$ respectively (Figure 5).

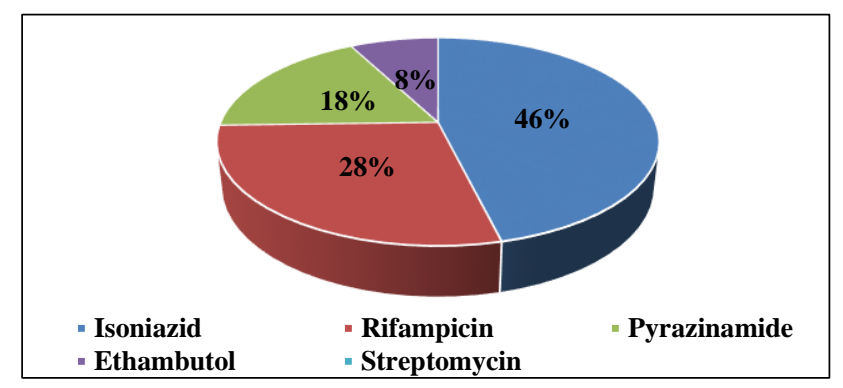

Figure 5: Total number of ADRs occurring due to individual drugs.
According to RNTCP category of TB wise distribution of ADRS, majority of ADR 33 (53\%) found in category II, 29 $(46 \%)$ were found in category I patients (Table 4).

Table 4: RNTCP category wise distribution of ADR involving different systems.

\begin{tabular}{|llll|}
\hline ADR & Category-I & Category-II & Total \\
\hline GIT & 16 & 12 & 28 \\
\hline Skin & 4 & 6 & 10 \\
\hline Musculoskeletal & 6 & 4 & 10 \\
\hline Hepatobiliary & 0 & 3 & 3 \\
\hline CNS and PNS & 3 & 3 & 6 \\
\hline Others & 0 & 5 & 5 \\
\hline Total & $29(46.77 \%)$ & $33(53.22 \%)$ & $62(100 \%)$ \\
\hline
\end{tabular}

\section{DISCUSSION}

Since, there can be hope to minimization of the adverse effects of drugs it was necessary to evaluate pattern of adverse reactions. There is a special need for systemic collection of information on ADRs in India due to wide variation in genetic, nutritional, environmental and disease patterns. Therefore, better approaches must be devised for reporting assessment and management of individuals who present with drug induced disease.

This study was planned for detection, assessment, classification and causality analysis of ADRs of tubercular patient attending at DOTS Center in Dr. Bhim Rao Ambedkar Memorial Hospital, Raipur, Chhattisgarh, India.

Total number of patients who were taking DOTS therapy in OPD (716) and in IPD (100). 62 ADRs detected was $7.5 \%$ was similar to a study Dhingra VK et al, observed $8.37 \%$ and Marra F et al, found 7.3\% ${ }^{10,11}$ Out of 62 ADRs, 26 ADRs were due to H, 16 ADRs were due to R, 10 ADRs were due to $\mathrm{Z}$ and 7, 3 ADRs due to $\mathrm{E}$ and $\mathrm{S}$ were observed respectively. ADRs reported by treating specialist and resident doctor and other health professionals of Chest Tuberculosis Department and DOTS Center Dr. Bhim Rao Ambedkar Memorial Hospital, Raipur, Chhattisgarh, India during the study period from 1 August 2011 to 31 July 2012. The information thus gathered about ADRs was compiled and analyzed in Department of Pharmacology.

The result of this study showed that the most common age of patient was 21-30 years followed by 31-40 years. However, a study conducted by Daphne Y et al, showed that most ADRs occur in patients above the age of 60 years and a study conducted by Tak DK et al, showed majorities of ADR were observed in patients with ages below 60 years which was similar to this study. ${ }^{12,13}$ It could be because less number of patients with ages above 60 years was included in this study.

Most of the literature says that the female gender was the one of the predisposing factors for ADRs and also, a study conducted by Daphne $\mathrm{Y}$ et al, showed that the female 
gender is at a high risk of developing ADRs. ${ }^{13}$ Also same was reported by Sinha $\mathrm{K}$ et al, But in this study more number of ADRs were seen in males patients $35(56.45 \%)$ and it could be because more numbers of males were in this study. ${ }^{14}$

The majority of ADRs was moderate $33(53.22 \%)$ and mild $29(46.77 \%)$ and no sever ADR were reported, the mild ADRs required no treatment or simply modification in the doses of suspected drug while moderate ADRs were required discontinuation of suspected drug and switch over to other ant tubercular drug or symptomatic treatment of ADRs.

Maximum number of ADRS detected were gastritis 28 $(45 \%)$ follow by $10(16 \%)$ of rashes, $10(16 \%)$ of arthralgia, $3(4 \%)$ of hepatitis and $6(9 \%)$ of vertigo.

The most common ADRs was gastritis. Out of which 10 occurred within the first week, 8 and 5 occurred in $2^{\text {nd }}$ and $3^{\text {rd }}$ week respectively, whose occurrence was less as compared to that seen in the study by Dhingra et al, where it was around $53 \% .^{10}$ The second most common reaction was skin reactions $16 \%$. However, in a study conducted by Dhingra et al. It was found to be around $17 \%$. The third most common reaction observed were arthralgia $16 \%$, whose occurrence was nearly as compared to a study Dhingra et al, where it was found $11 \%$.

Vestibular symptoms such as vertigo and ototoxicity were noted in 3 patients (4\%) who were on cat-II (DOTs) regimen in the $5^{\text {th }}$ and $6^{\text {th }}$ week of treatment of hepatotoxicity were noted in 3 patients $(4 \%)$ in this study whose occurrence was more as compared to that found in the studies conducted by Dhingra et al, and Zierski $\mathrm{M}$ et al, where it was found to be around $1 \%$ and 3.6-4-6\% respectively. ${ }^{10,15}$

Peripheral neuropathy occurred in 6 patients and the predisposing factor was found to be alcoholism. Symptomatic treatment (pyridoxine $100 \mathrm{mg}$ ) was given and DOTS therapy was continued.

Other reactions observed within $2^{\text {nd }}$ and $3^{\text {rd }}$ week of treatment were flu like syndrome.

In a study conducted by Tak DK et al, found that a majority of cases $38.09 \%$ were due to more than one drug. Similarly, in this study, $45 \%$ ADR were due to multiple drug therapy. ${ }^{12}$

In the first four weeks, around 95\% of the ADRs occurred in this study Dhingra et al, and Tak DK et al, reported in first four week $67 \%$ and $76.18 \%$ respectively. ${ }^{10,12}$

Causality assessment using standard method is probably the best way to establish the causal relationship between a drug and its effects. The Naranjo algorithm is used widely in the causality assessment of ADRs, in this study author found a majority of the ADRs to be "possible".
Present study revealed $46.77 \%$ mild and $53.22 \%$ moderate, there was $51 \%$ of type A reaction and $48 \%$ of type B reaction. There were $11 \%$ certain (definite) $35.48 \%$ probable and $53.22 \%$ of possible probability of ADRs identified by Naranjo causality scale.

\section{CONCLUSION}

Despite all limitations of under reporting biased reporting and missing data, this study was an attempt to generate more systemic knowledge about ADRs to anti-tubercular drugs of DOTS with the ultimate aim of doing something good for human beings.

\section{Funding: No funding sources}

Conflict of interest: None declared

Ethical approval: The study was approved by the Institutional Ethics Committee

\section{REFERENCES}

1. Devi S, Ramchandran R, Santha S. Adverse reaction to antituberculosis drugs and their management. Bulletin. 1997;4(3and4):1-4.

2. Tandon RK, Garg PK. Antituberculosis treatment induced hepatotoxicity. Sharma SK, Mohan, Tuberculosis. New Delhi: Jaypee Brothers; 2004:500.

3. Stork CM. Toxicology of antituberculosis drugs. Tubercul. 1996: 829-36.

4. National Tuberculosis control programmes including DOTS in Revised National Tuberculosis Control program (RNTCP): DOTS Stretegy including dots plus $3^{\text {rd }}$ Ed., 2001. New Delhi Century publications; 2001:195.

5. Tuberculosis and poverty. ICMR Bulletin. March 2002;32(3):25-32.

6. Styblo K. Tuberculosis control and surveillance. Recent Adv Resp Med. 1986;4:77-108.

7. Parthsarathi G, Gogtay N. Causality assessment of adverse drug reactions. Proceedings of the National Training Course on Pharmacovigilance. Mumbai; 2005:17-21.

8. Protocol for National Pharmacovigilance Programme Verson 1; Central Drug Standard Control Organization. Directorate General of Health Services, Ministry of Health and Family Welfare, Govt. of India. Nov 2004:87-115.

9. Bennett PN, Brown MJ, eds. Clinical Pharmacology. 9th ed. New York.

10. Dhingra VK, Rajpal S, Aggarwal N, Aggarwaln JK, Shadab K, Jain SK. Adverse drug reactions observed during DOTS. J Comm Dis. 2004;36(4):251-9.

11. Marra F, Marra CA, Bruchet N, Richardson K, Moadebi S, Elwood RK, et al. Adverse drug reactions associated with first-line anti-tuberculosis drug regimens. Inter J Tubercul Lung Dis. 2007;11(8):86875.

12. Tak DK, Acharya LD, Gowrinath K, Rao PGM, Subish P. Safety evaluation of antitubercular therapy 
under revised national tuberculosis control programme in India. J Clin Diagn Res. 2009;3(2):1395-401.

13. Daphne Y, Chantal V, Marthe P. Parisien Isabelle, Rocher Isabelle, Menzies Dick. Incidence of serious side effects from first-line antituberculosis drugs among patients treated for active tuberculosis. Am J Resp Crit Care Med. 2003;167:1472-7.

14. Sinha K, Marak IT, Singh WA. Adverse drug reactions in tuberculosis patients due to directly observed treatment strategy therapy: experience at an outpatient clinic of a teaching hospital in the city of Imphal, Manipur, India. J Assoc Chest Physicians. 2013;1(2):50.
15. Zierski M, Bek E. Side-effects of drug regimens used in short-course chemotherapy for pulmonary tuberculosis. Controlled Clin Study. Tubercl. 1980;61(1):41-9.

Cite this article as: Rao A, Ahmed T, Khan TA, Chandel NB, Mishra C. A study of adverse drug reaction profile of tuberculosis patients attending DOTS center at Dr. Bhim Rao Ambedkar memorial hospital, Raipur, Chhattisgarh, India. Int J Basic Clin Pharmacol 2019;8:662-8. 